\title{
Cost effectiveness of influenza vaccination for specific populations in Iran
}

\author{
Sanaei $Z^{1}$, Moradi-Lakeh $\mathbf{M}^{2}$, Kheiltash $A^{3}$, Amiri $\mathbf{J}^{4}$, Zahraei $\mathbf{S M}^{5}$, Yaghoubi $\mathbf{M}^{6^{*}}$ \\ ${ }^{1}$ Community Medicine Specialist, Department of Community Medicine, Education Development Office, Hamadan University of \\ Medical Science, Hamadan, Iran. \\ ${ }^{2}$ Community Medicine, Preventive Medicine and Public Health Research Center, Department of Community Medicine, Iran \\ University of Medical Sciences, Tehran, Iran. \\ ${ }^{3}$ Department of community and preventive medicine, School of medicine, Tehran University of Medical Science, Tehran, Iran. \\ ${ }^{4}$ Deputy for Health, Department of Pediatrics, Hamadan University of Medical Science, Hamadan, Iran. \\ ${ }^{5}$ Center for Communicable Disease Control, Ministry of Health and Medical Education, Tehran, Iran. \\ ${ }^{6}$ Clinical Knowledge Management Unit, Dept of Community Medicine, Iran University of Medical Science, \\ Tehran, Iran.
}

\begin{abstract}
Introduction: Influenza viruses are the major respiratory pathogens worldwide and high-risk groups such as healthcare workers may develop severe forms of the disease. The purpose of this study was to evaluate the cost-effectiveness of influenza vaccination for 4 target groups including pregnant women, elderly people (aged over 65 years), healthcare workers and school-age children in Iran. Methods: A cost-effectiveness analysis using a decision tree model over a one-year time horizon for the influenza vaccination versus no vaccination in Iran was carried out according to the prospective of the Ministry of Health. Epidemiological data were extracted from the relevant local databases and the literature. The medical and community care costs with sampling of the patients in all 4 groups were estimated. Results: The results of the economic evaluation showed that in Iran, incremental costs per Disability-Adjusted-Life-Years (DALY) of influenza were estimated to be US\$ 15,069 , US\$ 104,104, US\$ 5,685 and US\$ 14,983 for the pregnant women, the elderly people, the healthcare workers and the school-age children, respectively. Conclusion: The results of this study indicated that the implementation of influenza vaccination program might be cost effective only for the healthcare workers.
\end{abstract}

KEYWORDS: Influenza, cost-effectiveness, Iran, school-age children, pregnant women, elderly, healthcare workers.

\section{INTRODUCTION}

Influenza viruses $\mathrm{A}$ and $\mathrm{B}$ are the major respiratory pathogens in the world which cause epidemics, especially during the winter which can cause sporadic cases and outbreaks while influenza A virus can cause pandemics. Trivalent Influenza Vaccine (TIV) is the only vaccine which is licensed to be used for children from 24 months to 6 years, pregnant women and people over 50 years of age. When the vaccine strains match perfectly with the circulating virus, the efficacy of the vaccine in people under 65 years of age is 70-90\% [1]. High-risk groups in this regard are healthcare workers as well as other groups with a particular risk for developing severe forms of the disease which may lead to hospitalization or even death. The other concerned groups are pregnant women, children under 5 years

*Corresponding Author: Mohsen Yaghoubi, Clinical Knowledge Management Unit, Dept of Community Medicine, Iran University of Medical Science, Tehran, Iran

Email: mohsenyaq@yahoo.com

Tel/Fax: (+98) 2188602225/ (+98) 2188602217 of age, the elderly and people with debilitating diseases such as AIDS, asthma and chronic cardiopulmonary diseases. Groups at high-risk of developing influenza complications have received less attention in countries with low and middle income [1]. A world-wide analysis of the burden of different diseases in 2010 has shown that 4 causes of death include ischemic heart disease, ischemic stroke, chronic obstructive pulmonary disease and lower respiratory tract infections [2]. Influenza is a public health problem and has important implications for the healthcare systems due to an increase in the number of inpatient and out-patient clients. The socio-economic burdens of influenza are secondary to impaired quality of life and loss of productivity which all lead to increased healthcare costs either directly or indirectly [3]. Prior to the introduction of a drug or a vaccine, the healthcare authorities in many countries need to assess the cost-effectiveness of such interventions, especially when they require public subsidies or funding [4]. Having specific information from a country about its risk groups, disease burden and the cost-effectiveness of interventions are 
important for the health policy makers at national level to enable the authorities to choose specific populations for vaccinations [1]. The aim of this study was to estimate the costeffectiveness of public influenza vaccination program in Iran as a major population center in West Asia.

\section{MATERIALS and METHODS}

\section{Model structure}

A deterministic decision-tree model was used to compare 4 target populations with respect to influenza infection which included pregnant women, the elderly (over 65 years of age),

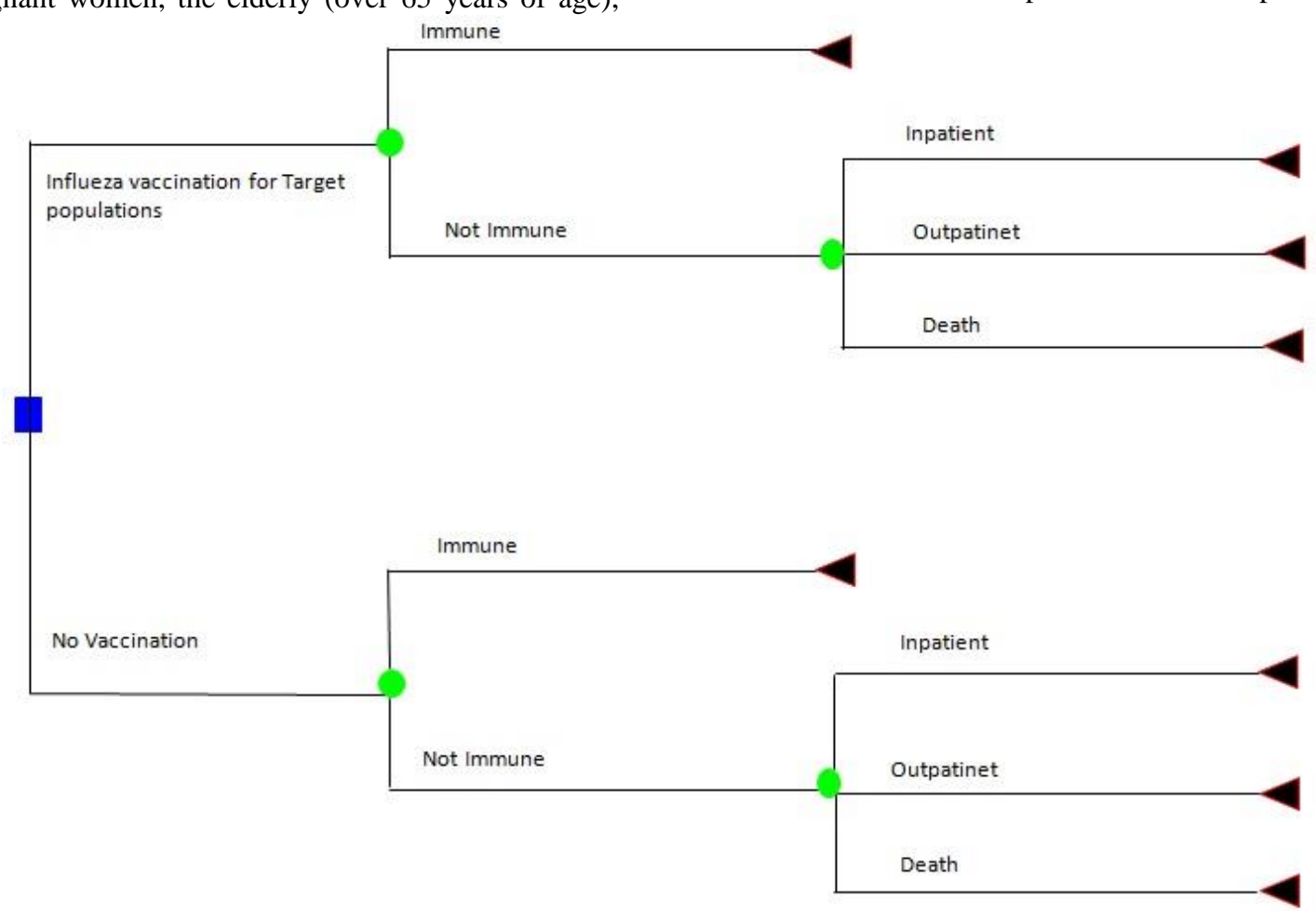

Fig. 1. Decision tree model to assess cost-effectiveness of influenza vaccination program.

The outcome was estimated as a total number of influenza cases averted, as well as Disability-Adjusted-Life-Years (DALYs) averted due to vaccination in each target population. The total costs of the implementation of vaccination program in the intervention scenarios, as well as the direct costs of disease management in non-vaccination scenarios were calculated based on the Ministry of Health and Medical Education prospective. By entering all epidemiological data, the costs and the vaccine efficacy into the model, the cost per DALY averted and the costs per averted cases were then calculated for each scenario. All costs were converted from Iranian Rials (IRR) into the United States Dollars (US\$), based on the average exchange rate in the base year of 2014 (1 US\$ $=30,000$ Rials).

\section{Parameter estimation}

Due to lack of epidemiological data from Iran, the required data were extracted from the Global Burden of Disease study (GBD 2013) $[2,5]$, conducted by the Institute for Health Metrics and Evaluation (IHME) to extract the incidences, deaths and DALYs due to influenza in Iran. For the school-age children, we extracted burden of disease parameters for children aged between 9 and 14 years and we considered the adults older than 65 years for the elderly people group. In order to extract data for the pregnant women, we assumed that pregnant women have a greater likelihood (about 4 fold) of getting influenza healthcare workers and school-age children based on data obtained from 2014 Ministry of Health and Medical Education prospective regarding vaccination in Iran. In this model, we followed the pathway from the original decision node (i.e. vaccination or no vaccination) to each of the terminal nodes. There were 2 distinct pathways including immune and not immune for the target populations who were not vaccinated as well as for whom the vaccination was not effective, based on the vaccination efficacy rate. We also considered probability of in-patient admissions, out-patient visits and deaths in each of the target populations throughout the decision tree model which was obtained from opinions of an expert panel (Fig. 1). 
meta-analysis. For the healthcare workers group, we calculated a pooled estimate of vaccine efficacy amongst 10 studies [8-17] for healthy adults aged 18-49 and results of the meta-analysis showed that pooled estimate of vaccine efficacy was equal to 66\% (CI: 60-72) and there was heterogeneity between the results of the studies $(\mathrm{Q}=13.69, \mathrm{P}=0.250)$. Regarding the school-age children, 2 studies [18, 19] were included and pooled estimate of the vaccine efficacy was $64 \%$ (CI: 51-76). There was no heterogeneity between the results of the studies $(\mathrm{Q}=3.15, \mathrm{P}=0.98)$. Pooled estimate of the vaccine efficacy for the elderly was estimated at $19 \%$ (CI: 10-27) between 2 included studies $[20,21]$ and there was no heterogeneity between the results of the studies $(\mathrm{Q}=5.895, \mathrm{P}=0.552)$. The vaccine efficacy for the pregnant women was equal to $43 \%$ (CI: 29-56) [22]. (Table 1)

\section{Vaccination costs}

The vaccine's price per dose was taken from the local representative of the vaccine's manufacturer (Jahan Behbood Corporation, Iran) that was US\$ 10 per dose. We added $3 \%$ surcharge of purchasing from the manufacturer to the price of the vaccine. In addition, $2 \%$ was added for transportation, including the insurance, shipping and delivery to the airport. Also 5\% wastage rate was assumed, based on the expert opinion for the vaccine. To estimate the vaccine supplies costs, the following formula was used:

$\mathrm{C}=\mathrm{P} * \mathrm{I} \times \mathrm{B} \times \mathrm{D} \times(1 /(1-\mathrm{w})$

$\mathrm{P}=$ Vaccine price per dose

I = Immunization coverage rate

$\mathrm{B}=$ Birth cohort

$\mathrm{D}=$ Number of doses per fully immunized child

$\mathrm{W}=$ Wastage rate

The number of doses per children fully immunized for influenza vaccine, the pregnant women, the elderly (aged > 65 ) and the healthcare workers was considered as 1 dose while for school-age children (aged < 9) 2 doses were assumed. We estimated that the number of Iranian pregnant women in the cohort to be 1,380,000 in the base year. The elderly population was 4,296,769 and the cohort of healthcare workers in the base year was assumed to be 500,000 . The total population of school-age children was considered to be $4,437,473$. We assumed $99 \%$ coverage rate of the vaccination. Consequently, the total vaccine supplies for the pregnant women, the elderly population, the healthcare workers and the school-age children were US\$ $14,381,053$, US\$ 44,664,914, US\$ 5,197,500 and US\$ 92,255,064, respectively. We used World Health Organization (WHO) guideline to estimate the incremental costs of introducing a new vaccine into the current national vaccination system. To estimate the incremental system cost per dose, we included the costs of cold-chain, surveillance, monitoring, training, maintenance and the required facilities which are needed beyond the currently available facilities of Iran's Ministry of Health and Medical Education. The total annualized capital cost was estimated based on equipment prices and their useful life and an annualizing factor (Table 2).

\section{Health services costs}

The direct medical costs of treatment in each of the 4 target groups used for management of influenza were estimated for inpatient and out-patient cases, using sampling done at Imam Khomeini Hospital in Tehran which is the main hub of the influenza treatment for outbreak cases. For this purpose, 20 cases of the hospital treatments for each of the above 4 groups were studied and the average cost of hospitalization, diagnostic tests costs, medications and GP visits were collected and calculated. Furthermore, to calculate the costs for out-patient services, we extracted pharmaceutical items, diagnostic tests and out-patient visits after consultation with an expert's panel including health professionals, GPs, pediatric assistants and pediatricians in public hospitals and estimated the average costs per out-patient visits. (Table 2)

Cost-effectiveness analysis

The analysis of the cost effectiveness of vaccination against influenza of Iranian Ministry of Health and Medical Education's prospective was compared to no vaccination. To estimate the incremental cost-effectiveness ratio (ICER), the difference between the costs in the 2 scenarios was divided by the difference of DALYs averted according to the following formula[23] and WHO threshold was used to interpret the incremental cost-effectiveness ratio.

ICER $=($ Total Vaccination Cost - Medical Cost Saved $) /$ (DALY without vaccine - DALY with vaccine)

Sensitivity Analysis

A one-way sensitivity analysis was performed on each scenario to detect those parameters which had the most impact on the ICER. We considered upper and lower estimates of DALYs, inpatient and out-patient health services costs and the vaccine efficacy in this regard.

Table 1. Summary of model parameters

\begin{tabular}{|c|c|c|c|c|}
\hline Parameter & Pregnant Women & Elderly people & Heath care workers & School age children \\
\hline Number of Cohort & $1,380,000$ & $4,296,769$ & 500,000 & $4,437,473$ \\
\hline Incidence per 100 & 3.4 & 12.4 & 0.9 & 1.4 \\
\hline Influenza cases & 47,472 & 532,799 & 4,950 & 62,125 \\
\hline Inpatient cases & 2,673 & 148,575 & 291 & 1,722 \\
\hline Outpatient cases & 44,799 & 384,202 & 4,659 & 60,402 \\
\hline Death & $30.2(18.2-44.4)$ & $125(71.2-180.1)$ & $22.5(12.8-31.5)$ & $7.7(4.81-10.41)$ \\
\hline DALY & $2059(1404-2635)$ & $1576(951-2315)$ & $1353(772-2213)$ & $971(605-1541)$ \\
\hline Average Inpatient cost & US\$400(\$129.5-\$670.6) & US\$333(\$62.5-\$603.5) & US\$488(\$217.7-\$758.7) & US\$113(\$53.26-\$313.7) \\
\hline Average Outpatient cost & US\$39.3(\$33.4-\$45.1) & US\$39.3(\$33.4-\$45.1) & US\$39.3(\$33.4-\$45.1) & US\$38.4(\$32.5-\$44.2) \\
\hline Vaccine efficacy & $0.43(0.29-0.56)$ & $0.19(0.10-0.27)$ & $0.66(0.60-0.72)$ & $0.64(0.51-0.76)$ \\
\hline
\end{tabular}


Table 2. Vaccine cost parameters

\begin{tabular}{|c|c|c|c|c|}
\hline Parameter & Pregnant Women & Elderly people & Heath care workers & School-age children \\
\hline Vaccine Price per dose & US\$10 & US\$10 & US $\$ 10$ & US\$10 \\
\hline Wastage factor & 1.05 & 1.05 & 1.05 & 1.05 \\
\hline Coverage rate \% & 0.99 & 0.99 & 0.99 & 0.99 \\
\hline Total dose required & $1,483,105$ & $4,466,491$ & 519,750 & $9,225,506$ \\
\hline $\begin{array}{c}\text { Total vaccine supplied } \\
\text { Cost } \\
\end{array}$ & US\$14,381,053 & US $\$ 44,664,914$ & US $\$ 5,197,500$ & US\$92,255,064 \\
\hline Cold chain storage & US $\$ 21,316$ & US $\$ 21,316$ & US\$21,316 & US\$21,316 \\
\hline Syringe and safety box & US $\$ 128,800$ & US $\$ 401,030$ & US $\$ 40,000$ & US $\$ 1,124,146$ \\
\hline Training material & US\$33,333 & US\$33,333 & US $\$ 33,333$ & US\$33,333 \\
\hline Surveillance & US\$23,333 & US\$23,333 & US\$23,333 & US\$23,333 \\
\hline Redesign of stationery & US\$7,666 & US\$7,666 & US\$7,666 & US\$7,666 \\
\hline Transport maintenance & US\$6,667 & US\$6,667 & US\$6,667 & US\$6,667 \\
\hline Cold chain maintenance & US $\$ 20,000$ & US $\$ 20,000$ & US $\$ 20,000$ & US\$20,000 \\
\hline Wastage Management & US $\$ 9,200$ & US $\$ 9,200$ & US $\$ 9,200$ & US\$9,200 \\
\hline $\begin{array}{l}\text { Total Logistic Cost } \\
\end{array}$ & US\$250,316 & US\$522,526 & US $\$ 108,483$ & US $\$ 2,558,674$ \\
\hline Incremental cost per dose & US\$0.17 & US\$0.11 & US\$0.20 & US\$0.27 \\
\hline
\end{tabular}

\section{RESULTS}

The average cost of out-patient treatments for influenza was estimated to be US\$38.4 for the school-age children while this amount was US\$ 39.3 for the other 3 population groups. The cost of in-patient admissions totaled US\$ 400 for the pregnant women, US\$ 333 for the elderly and US\$ 488 for the healthcare workers and US\$ 113 for the school-age children in Ministry of Health prospective (Table 1). Total cost of influenza disease management for the pregnant women, the elderly, the healthcare workers and the school-age children were US\$ 2,995,093 US\$ 73, 731,567, US\$ 343,351 and US\$ 2,621,466, respectively in no vaccination scenario for the base year.

For the pregnant women population, total number of deaths averted was 13.8 and the numbers of influenza cases avoided were 20,413. Furthermore, total costs of influenza saved were calculated to be US\$1,287,890 during the base year. In the elderly population, total number of influenza cases averted was 101,232 and number of deaths averted was 23.89 , total cost of influenza management averted was obtained at US\$
$14,008,998$. For the healthcare worker population the total number of deaths averted was at 14.9 and the number of influenza cases avoided was 3,267. Moreover, the total cost of influenza saved was US\$226,612 during the base year. In the school-age population, the total number of influenza cases averted was 39,760 and the number of deaths averted was 4.9. The total cost of influenza management saved was calculated to be US\$1,677,738 (Table 3).

Incremental cost per DALY averted for vaccination program compared to no vaccination for the target groups of the pregnant women, the elderly people, the healthcare workers and the school age children was estimated to be US\$15,069, US\$ 104,104 , US\$ 5,685 and US\$ 149,839, respectively (Table 3). Results of the sensitivity analysis showed that ICER for the pregnant women vaccination program was changed from US\$ 11,233 to US\$23,045. This value changed from of US\$59,399 to US\$239,897 for the elderly group. Also for the healthcare workers group, the ICER results changed from US\$ 3,578 to US\$ 99, 69 and for the school-age children program, this value changed from US\$ 94,435 to US\$240,537 (Fig. 2).

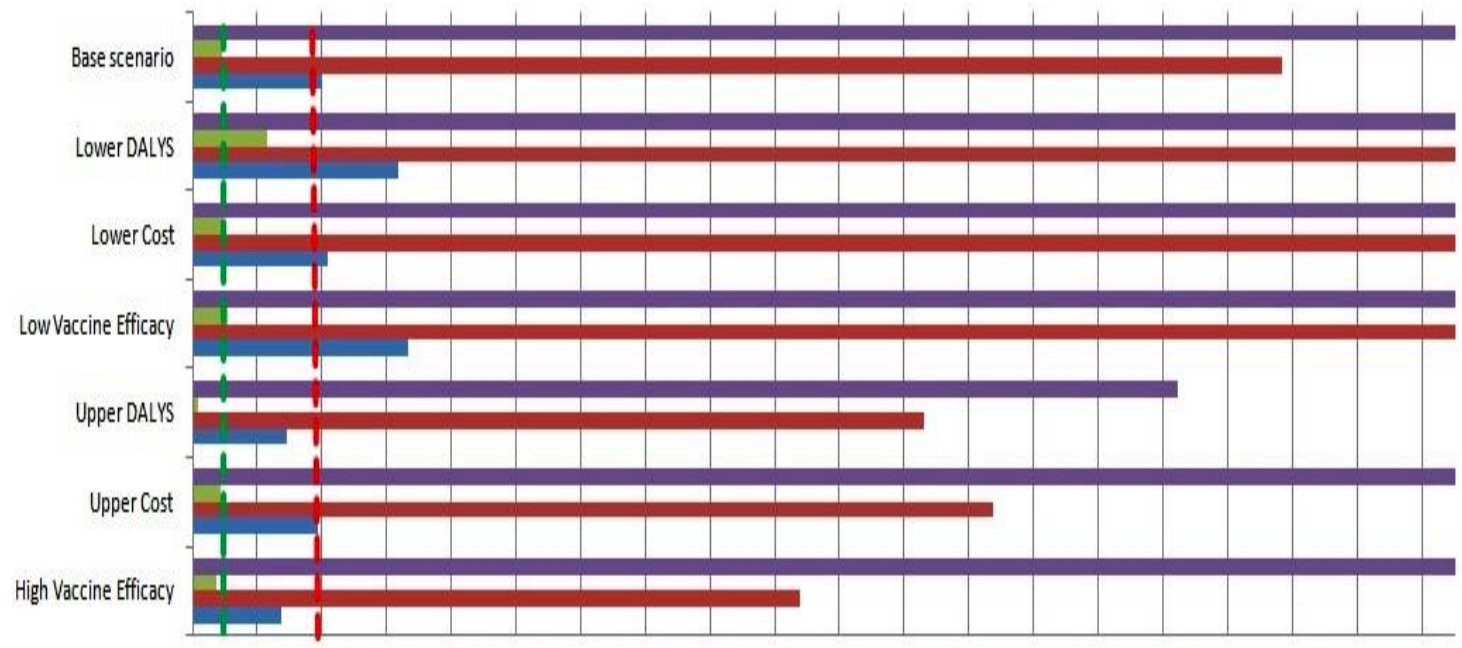

WChilderen

Health care worker

IElderly

upergnant

$3,000 \quad 9,000 \quad 15,00021,00027,000 \quad 33,000 \quad 39,000 \quad 45,000 \quad 51,000 \quad 57,000 \quad 63,000 \quad 69,000 \quad 75,00081,000 \quad 87,00093,00099,000$ 105,000111,000117,000

Fig. 2. Scenario analysis of ICER in influenza vaccination program for 4 different Iranian populations studied. 
Table 3. Total estimated costs, healthcare utilization measures and cost- effectiveness results.

\begin{tabular}{|c|c|c|c|c|}
\hline Item & Pregnant Women & Elderly people & Heath care worker & School age children \\
\hline Total Vaccination cost & US\$14,631,369 & US\$45,187,460 & US\$5,305,983 & US $\$ 94,813,739$ \\
\hline Medical and community care cost & $2,995,093$ & $73,731,567$ & 343,351 & $2,621,466$ \\
\hline Medical and community care cost saved & $1,287,890$ & $14,008,998$ & 226,612 & $1,667,738$ \\
\hline Number of case averted & 20,413 & 101,232 & 3,267 & 39,760 \\
\hline Number of Inpatient admission averted & 1,149 & 28,229 & 192 & 1,102 \\
\hline Number of outpatient visit averted & 19,263 & 72,998 & 3,075 & 38,675 \\
\hline Number of death averted & 13 & 23 & 14 & 4.9 \\
\hline DALY averted & 885 & 299 & 893 & 621 \\
\hline Incremental cost per DALY averted & $\begin{array}{c}\text { US\$ } 15,069 \\
\text { Not } \\
\text { cost-effective }\end{array}$ & $\begin{array}{c}\text { US\$ } 104,104 \\
\text { Not } \\
\text { cost-effective }\end{array}$ & $\begin{array}{c}\text { US\$ 5,685 } \\
\text { Cost-effective }\end{array}$ & $\begin{array}{c}\text { US\$ } 149,839 \\
\text { Not } \\
\text { cost-effective } \\
\end{array}$ \\
\hline
\end{tabular}

\section{DISCUSSION}

According to the threshold of WHO as well as the incremental cost per DALY averted with the basis of per capita GDP in Iran (US\$ 4,763 in 2013), and the results of this study, it can be concluded that implementation of influenza vaccination program, only for the healthcare workers will be cost effective and the vaccination programs for the pregnant women, the elderly people and the children of school-age will not be cost effective, given that the incremental cost-effectiveness of such interventions is more than 3 times of the national GDP. Based on the sensitivity analysis, ICER for the healthcare workers group was still cost-effective (less than 3 time of the GDP per capita) in all scenarios. Moreover, in the upper scenario for DALYs, ICER for this group showed highly cost effective intervention $(\$ 3,487)$. However, a vaccination program for the pregnant women will be cost-effective in the upper scenario for DALYs $(\$ 11,777)$ and the upper scenario for the vaccine efficacy $(\$ 11,233)$. Results for other target groups remained constant in terms of cost-effectiveness (Fig. 2).

Regarding the incidence of influenza in the healthcare workers population, there was no significant difference between this group and the other 3 groups. However, due to low population of this group, and lower costs of implementation of vaccination program, the vaccination will be cost effective in this group. The vaccination of the pregnant women, the children of schoolage and the elderly people was not shown to be cost-effective due to the high costs of the vaccination program as well as their relatively low incidence.

Among 8 studies, all conducted in high-income countries where the economic evaluation of seasonal vaccination and influenza pandemic were examined, 7 studies were on elderly people in which 4 studies [24-27] evaluated the cost effectiveness for the seasonal influenza vaccine, and 2 studies $[25,28]$ evaluated the cost-effectiveness of influenza vaccines in addition to pneumococcal vaccine, compared to influenza vaccination alone, and one study [29] assessed the influenza vaccination for pregnant women. One study which has conducted in several countries has evaluated vaccination coverage in terms of gross national income per capita and examined the effects of increased income and education on immunization coverage[30]. Two studies have considered the factors affecting the cost effectiveness of the vaccine before and during the pandemic including the vaccine's strain match, the vaccine's availability and its cost [24, 31]. These studies are discussed in detail as follows.

The study of cost effectiveness of influenza vaccine in Australia, on people aged between 50 to 64 years has shown that vaccination programs in this age group reduced the annual incidences of Influenza-Like-Illness (ILI) to 3,124 cases, in fact, it is considered as $0.09 \%$ reduction in the incidence of ILI (reduced from $1.90 \%$ to $1.81 \%$ ). This new policy prevented 1,172 hospitalizations cases, 89 deaths and 2,805 cases of absenteeism from work. From the prospective of the healthcare payers and the government, the incremental costs of influenza vaccination program for years with perfect quality obtained, were US\$ 8,908, US $\$ 8,338$ and US $\$ 22,408$, respectively [27]. Also, another study have shown that the combined influenza and pneumococcal vaccination strategy has higher effectiveness than influenza vaccination alone [28].

In Japan, following rapid implementation of a national immunization program of influenza vaccination of the elderly, a study was conducted to review the efficiency of the implemented strategies in order to allocate allowances to their vaccination programs. The results indicated that a strategy that pays $100 \%$ allowances for all, or $100 \%$ allowance for the highrisk elderly, would be cost-effective [25]. In Australia, a model was used to compare the cost-effectiveness of universal influenza vaccination for persons over 50 years of age with recent policies of vaccination of people older than 65 years. According to the available epidemiological data, 2 scenarios were followed. One scenario was related to an estimation of the incidence of ILI in Australia, and the other one in Europe. These scenarios and sensitivity analyses have shown that, ICER compared to the recent policy indicate changes from US\$6,000 to US\$12,000 per DALYs [26].

Another study has examined the cost- effectiveness of influenza vaccination in pregnant women and has shown that, immunization of pregnant women population against influenza would save US\$ 50 per woman, gaining 45 quality-adjusted hours, relative to providing supportive care only. This study has determined that influenza vaccination programs for pregnant women are cost-effective [29]. Another study in Hong Kong aimed to answer the question "Does the program of influenza vaccination during a non-pandemic rate (with ILI value of $11 \%$ ), has the cost-benefit or not?" The results indicated that from the perspective of a susceptible individual, the influenza vaccine was cost-effective [24]. In a study in Singapore, a decision analytic model to assess the cost-benefit and cost effectiveness of vaccination against pandemic influenza was used. This model has compared common strategies for the treatment, including quick treatment with oseltamivir and only treatment with vaccination in addition to the treatment. According to their results, the only treatment strategy led to 690 deaths, 13,950 hospitalization days, and a cost of about US\$ 497 million in North America. For immediate vaccination, when vaccine efficiency was $55 \%$, the cost benefit of vaccination was more than vaccination alone. Stockpiling of the vaccines in most scenarios, even when the vaccine effectiveness 
is $100 \%$, is not cost-effective. Based on findings of this study, a vaccination program should be based on severity of the disease and immediate vaccination is most cost-effective; however, it implies that the vaccines be available when required [32]. Furthermore, results of a survey done in 10 countries in Africa, Asia, Oceania, Eastern Europe, Latin America and the Middle East have shown that influenza vaccination coverage rates between countries have considerable differences, and only 1 factor does not make the top cover. The results have indicated that a strong recommendation to perform vaccination is not enough, and the immunization programs will be completed when awareness of the population is increased. In fact, increased awareness is the key to increased coverage of influenza vaccination [30].

The authors here recommended that national immunization program in Iran should be focused on diseases with high morbidity and mortality, including Haemophilus Influenzae type b (Hib) infection, diarrhea and other severe infectious diseases based on economic evaluation studies which has been done in this regard [33-35]. In case of influenza, vaccination of high-risk group such as healthcare workers should be taken into consideration.

\section{ACKNOWLEDGEMENT}

The authors would like to thank all advisors, especially Dr. Susan Mahmoud from Center for Communicable Disease Control in Ministry of Health and Medical Education.

\section{CONFLICT OF INTEREST}

The authors declare that they have no conflict of interest.

\section{REFERENCES}

1. World Health Organization. "Vaccines against influenza WHO position paper-November 2012." Wkly Epidemiol Rec 87, no. 47(2012): 461-76.

2. Lozano R, Naghavi M, Foreman K, Lim S, Shibuya K, Aboyans V et al. Global and regional mortality from 235 causes of death for 20 age groups in 1990 and 2010: a systematic analysis for the Global Burden of Disease Study 2010. The lancet. 2012 Dec 15;380(9859):2095-128.

3. Zuccotti GV, Fabiano V. Influvac, a trivalent inactivated subunit influenza vaccine. Expert opinion on biological therapy. 2011 Jan 1;11(1):89-98. doi:10.1517/14712598.2011.541436.

4. Aldrich R, Kemp L, Williams JS, Harris E, Simpson S, Wilson A et al. Using socioeconomic evidence in clinical practice guidelines. BMJ: British Medical Journal. 2003 Nov 29;327(7426):1283.

5. Murray CJ, Vos T, Lozano R, AlMazroa MA, Memish ZA. Disabilityadjusted life years (DALYs) for 291 diseases and injuries in 21 regions, 1990-2010: a systematic analysis for the Global Burden of Disease Study 2010 (vol 380, pg 2197, 2012).

6. Siston AM, Rasmussen SA, Honein MA, Fry AM, Seib K, Callaghan WM et al. Pandemic 2009 influenza A (H1N1) virus illness among pregnant women in the United States. Jama. 2010 Apr 21;303(15):1517-25.

7. Williams CJ, Schweiger B, Diner G, Gerlach F, Haaman F, Krause G et al. Seasonal influenza risk in hospital healthcare workers is more strongly associated with household than occupational exposures: results from a prospective cohort study in Berlin, Germany, 2006/07. BMC infectious diseases. 2010 Jan 12;10(1):8. doi:10.1186/1471-2334-10-8.

8. Barrett PN, Berezuk G, Fritsch S, Aichinger G, Hart MK, El-Amin W et al. Efficacy, safety, and immunogenicity of a Vero-cell-culture-derived trivalent influenza vaccine: a multicentre, double-blind, randomised, placebo-controlled trial. The Lancet. 2011 Mar 4;377(9767):751-9. doi:10.1016/S0140-6736(10)62228-3.

9. Beran J, Vesikari T, Wertzova V, Karvonen A, Honegr K, Lindblad N et al. Efficacy of inactivated split-virus influenza vaccine against cultureconfirmed influenza in healthy adults: a prospective, randomized, placebocontrolled trial. The Journal of infectious diseases. 2009 Dec
15;200(12):1861-9. doi:10.1086/648406.

10. Beran J, Wertzova V, Honegr K, Kaliskova E, Havlickova M, Havlik J et al. Challenge of conducting a placebo-controlled randomized efficacy study for influenza vaccine in a season with low attack rate and a mismatched vaccine B strain: a concrete example. BMC infectious diseases. 2009 Jan 17;9(1):2. doi:10.1186/1471-2334-9-2.

11. Frey S, Vesikari T, Szymczakiewicz-Multanowska A, Lattanzi M, Izu A, Groth N et al. Clinical Efficacy of Cell Culture-Derived and Egg-Derived Inactivated Subunit Influenza Vaccines in Healthy Adults. Clinical Infectious Diseases. 2010 Nov 1;51(9):997-1004. doi:10.1086/656578.

12. Jackson LA, Gaglani MJ, Keyserling HL, Balser J, Bouveret N, Fries L et al. Safety, efficacy, and immunogenicity of an inactivated influenza vaccine in healthy adults: a randomized, placebo-controlled trial over two influenza seasons. BMC infectious diseases. 2010 Mar 17;10(1):71. doi:10.1186/1471-2334-10-71.

13. Monto AS, Ohmit SE, Petrie JG, Johnson E, Truscon R, Teich E et al. Comparative efficacy of inactivated and live attenuated influenza vaccines. New England Journal of Medicine. 2009 Sep 24;361(13):1260-7. doi:10.1056/NEJMoa0808652.

14. Ohmit SE, Victor JC, Rotthoff JR, Teich ER, Truscon RK, Baum LL et al. Prevention of antigenically drifted influenza by inactivated and live attenuated vaccines. New England Journal of Medicine. 2006 Dec 14:355(24):2513-22. doi:10.1056/NEJMoa061850.

15. Ohmit SE, Victor JC, Teich ER, Truscon RK, Rotthoff JR, Newton DW et al. Prevention of symptomatic seasonal influenza in 2005-2006 by inactivated and live attenuated vaccines. The Journal of infectious diseases. 2008 Aug 1;198(3):312-7. doi:10.1086/589885.

16. Sullender W, Fowler K, Krishnan A, Gupta V, Moulton LH, Lafond K et al. Design and initiation of a study to assess the direct and indirect effects of influenza vaccine given to children in rural India. Vaccine. $2012 \mathrm{Jul}$ 27;30(35):5235-9. doi:10.1016/j.vaccine.2012.06.002

17. Treanor JJ, El Sahly H, King J, Graham I, Izikson R, Kohberger R et al. Protective efficacy of a trivalent recombinant hemagglutinin protein vaccine (FluBlok $\left({ }^{\circledR}\right)$ against influenza in healthy adults: a randomized, placebocontrolled trial. Vaccine. 2011 Oct 13;29(44):7733-9. doi:10.1016/j.vaccine.2011.07.128.

18. Cowling BJ, Ng S, Ma ES, Fang VJ, So HC, Wai W et al. Protective efficacy against pandemic influenza of seasonal influenza vaccination in children in Hong Kong: a randomized controlled trial. Clinical Infectious Diseases. 2012 Jun 5;55(5):695-702. doi:10.1093/cid/cis518.

19. Ng S, Ip DK, Fang VJ, Chan KH, Chiu SS, Leung GM et al. The effect of age and recent influenza vaccination history on the immunogenicity and efficacy of 2009-10 seasonal trivalent inactivated influenza vaccination in children. PloS one. 2013 Mar 12;8(3):e59077.

20. DiazGranados CA, Dunning AJ, Jordanov E, Landolfi V, Denis M, Talbot HK. High-dose trivalent influenza vaccine compared to standard dose vaccine in elderly adults: safety, immunogenicity and relative efficacy during the 2009-2010 season. Vaccine. 2013 Jan 30;31(6):861-6. doi:10.1016/j.vaccine.2012.12.013.

21. McElhaney JE, Beran J, Devaster JM, Esen M, Launay O, Leroux-Roels $\mathrm{G}$ et al. AS03-adjuvanted versus non-adjuvanted inactivated trivalent influenza vaccine against seasonal influenza in elderly people: a phase 3 randomised trial. The Lancet infectious diseases. 2013 Jun 30;13(6):485-96. doi:10.1016/S1473-3099(13)70046-X.

22. Omer SB, Zaman K, Roy E, Arifeen SE, Raqib R, Noory L et al. Combined effects of antenatal receipt of influenza vaccine by mothers and pneumococcal conjugate vaccine receipt by infants: results from a randomized, blinded, controlled trial. The Journal of infectious diseases. 2013 Jan 8;207(7):1144-7. doi:10.1093/infdis/jit003.

23. Edejer TT, Baltussen R, Adam T, Hutubessy R, Acharya A, Evans DB et al. WHO guide to cost-effectiveness analysis.

24. Fitzner KA, Shortridge KF, McGhee SM, Hedley AJ. Cost-effectiveness study on influenza prevention in Hong Kong. Health Policy. 2001 Jun 30;56(3):215-34. doi:10.1016/S0168-8510(00)00140-8.

25. Hoshi SL, Kondo M, Honda Y, Okubo I. Cost-effectiveness analysis of influenza vaccination for people aged 65 and over in Japan. Vaccine. 2007 Aug 29;25(35):6511-21. doi:10.1016/j.vaccine.2007.05.067.

26. Mogasale V, Barendregt J. Cost $\square$ effectiveness of influenza vaccination of people aged 50-64 years in Australia: results are inconclusive. Australian and New Zealand journal of public health. 2011 Apr 1;35(2):180-6. doi: 10.1111/j.1753-6405.2010.00639.x.

27. Newall AT, Scuffham PA, Kelly H, Harsley S, MacIntyre CR. The costeffectiveness of a universal influenza vaccination program for adults aged 50-64 years in Australia. Vaccine. 2008 Apr 16;26(17):2142-53. doi:10.1016/j.vaccine.2008.01.051. 
28. You JH, Wong WC, Ip M, Lee NL, Ho SC. Cost-effectiveness analysis of influenza and pneumococcal vaccination for Hong Kong elderly in longterm care facilities. Journal of Epidemiology \& Community Health. 2009 Nov 1;63(11):906-11. doi:10.1136/jech.2008.081885.

29. Roberts S, Hollier LM, Sheffield J, Laibl V, Wendel Jr GD. Costeffectiveness of universal influenza vaccination in a pregnant population. Obstetrics \& Gynecology. 2006 Jun 1;107(6):1323-9. doi:10.1097/01.AOG.0000210225.45986.99.

30. Lataillade CD, Auvergne S, Delannoy I. 2005 and 2006 seasonal influenza vaccination coverage rates in 10 countries in Africa, Asia Pacific, Europe, Latin America and the Middle East. Journal of public health policy. 2009;30(1):83-101. doi:10.1057/jphp.2008.40.

31. Samaan G, McPherson M, Partridge J. A review of the evidence to support influenza vaccine introduction in countries and areas of WHO's Western Pacific Region. PloS one. 2013 Jul 16;8(7):e70003.
32. Lee VJ, Tok MY, Chow VT, Phua KH, Ooi EE et al. Economic analysis of pandemic influenza vaccination strategies in Singapore. PLoS One. 2009 Sep 22;4(9):e7108

33. Javanbakht M, Moradi-Lakeh M, Yaghoubi M, Esteghamati A, Ghanaie $\mathrm{RM}$, Mahmoudi S et al. Cost-effectiveness analysis of the introduction of rotavirus vaccine in Iran. Vaccine. 2015 May 7;33:A192-200. doi:10.1016/j.vaccine.2014.12.035.

34. Moradi-Lakeh M, Shakerian S, Esteghamati A. Immunization against Haemophilus influenzae type $\mathrm{b}$ in Iran; cost-utility and cost-benefit analyses. International journal of preventive medicine. 2012 May;3(5):332. 35. Shakerian S, Lakeh MM, Esteghamati A, Zahraei M, Yaghoubi M. CostEffectiveness of Rotavirus Vaccination for Under-Five Children in Iran Iranian journal of pediatrics. 2015 Aug;25(4). doi:10.5812/ijp.2766. 